\title{
Aporte de materia orgánica de Crotalaria spectabilis Roth, en un suelo sembrado con Brachiaria brizantha CV. BRS piatá
}

\author{
Emmanuel de Jesús Chaves González ${ }^{1}$, Paola Brenes Rojas ${ }^{2}$ \& Wagner Peña Cordero ${ }^{3}$ \\ 1. Ingeniero Agrónomo, Trabajo final de graduación UNED, emmachg09@gmail.com \\ 2. Vicerrectoría de Investigación UNED, paolarjs@gmail.com. \\ 3. Encargado de Catedra de gestión sostenible del Suelo Escuela de Ciencias Exactas y Naturales, UNED. \\ wpena@uned.ac.cr. \\ Universidad Estatal a Distancia. Apartado Postal 474-2050. San Pedro, San José. Costa Rica.
}

Recibido: 14 de abril de 2018

\section{RESUMEN}

El contenido óptimo de materia orgánica en los suelos es esencial para la nutrición de los forrajes, por eso ha sido necesario buscar alternativas para mejorar la nutrición de las pasturas, En esta investigación se analizó el efecto del rastrojo de Crotalaria spectabilis Roth, sobre las propiedades químicas del suelo. La investigación se llevó a cabo en el cantón de San Carlos, utilizando un diseño experimental factorial compuesto por tres tratamientos y tres repeticiones, evaluadas a los 0 y 88 días después de sembrar el pasto, al cual durante la siembra se le aplicó diferentes dosis del rastrojo de Crotalaria spectabilis Roth. Al concluir el experimento se evidenció un incremento en las variables evaluadas, debido a una mayor humificación del rastrojo incorporado y una liberación de nutrientes más rápida al suelo.

Palabras claves: Nitrógeno, Crotalaria, abono verde, Pasto Piatá.

\begin{abstract}
The optimum content of organic matter in the soils is essential for the nutrition of forages, that is why it has been necessary to look for alternatives to improve the nutrition of the pastures. In this investigation the effect of stubble of Crotalaria spectabilis Roth on the properties was analyzed. soil chemicals. The investigation was carried out in the San Carlos canton, using a factorial experimental design composed of three treatments and three repetitions, evaluated at 0 and 88 days after sowing the grass, which during the sowing was applied different doses of the. At the end of the experiment an increase in the evaluated variables was evidenced, due to a greater humification of the incorporated stubble and a faster release of nutrients to the soil.
\end{abstract}

Keywords: Nitrogen, Crotalaria, green manure, Piatá grass.
Aceptado: 12 de agosto de 2018

\section{Introducción}

Los pastos constituyen la principal fuente de nutrientes para la alimentación del ganado bovino, es el medio más económico y utilizado en las regiones tropicales y la fuente más barata para la alimentación de rumiantes. Para el establecimiento de pasturas, es necesaria la fertilización, que es una de las prácticas agronómicas más importantes. La fertilización de potreros en su etapa de establecimiento se enfoca en la aplicación de nitrógeno y fósforo, dicha práctica puede no resultar adecuada ya que parte del fertilizante puede perderse o fijarse en el suelo. Según Araya (2014) a partir de esto se vuelve necesaria la incorporación de nutrimentos mediante alternativas que garanticen la entrada de nutrientes limitantes al sistema.

A esta situación hay que añadir, que en Costa Rica los suelos destinados al cultivo de pastos en su mayoría son de baja fertilidad, baja disponibilidad de nitrógeno, $\mathrm{pH}$ ácidos, mal drenados, bajo contenido de materia orgánica, que en conjunto con el clima ejercen efectos negativos en la productividad, calidad y persistencia de las especies de pastos establecidas (Ramírez, Herrera, Leonard, Verdecia y Álvarez, 2012).

La materia orgánica es fundamental para mejorar las propiedades físicas, químicas y biológicas del suelo. La presencia de materia orgánica en el suelo, entre otras funciones, ayuda al desarrollo o mantenimiento del complejo arcillohúmico, fundamental para garantizar una buena 
movilidad de los nutrientes, contribuyendo a mantener un $\mathrm{pH}$ del suelo óptimo, fundamental para la asimilación de ciertos nutrientes en el suelo, además facilita el mantenimiento de una actividad microbiológica óptima, evita la pérdida de algunos nutrientes en el suelo a causa de lixiviación, volatilización, escorrentía, y lavado.

Según Durán (2015), como alternativa se deben de establecer producciones agrícolas que sean conservacionistas y tengan una mayor eficiencia y disponibilidad de nutrientes para los forrajes. Es por ello, por lo que se hace necesario el uso del rastrojo de leguminosas, como Crotalaria spectabilis Roth, que es un abono verde que aporta nitrógeno de forma natural, e incrementa los valores de materia orgánica mediante el proceso de fijación biológica. Esta estrategia se puede aplicar en los suelos de fincas ganaderas que han sido utilizadas por producciones previas y en donde se han aplicado fertilizantes de origen sintético para aumentar la disponibilidad de nitrógeno $(\mathrm{N})$, en el suelo (Arias, 2015). De esta forma las leguminosas contribuyen a la nutrición nitrogenada permitiendo un mayor aprovechamiento del nitrógeno y una mayor productividad de las praderas, e incrementando los valores de materia orgánica en el suelo (Paredes, 2013).

\section{Área de estudio}

La zona de estudio se ubicó en el cantón de San Carlos, cantón número 10 de la provincia de Alajuela, distrito la Fortuna, Poblado la Perla. El estudio se realizó en un suelo de orden ultisol proveniente del Centro de Investigación Transferencia Tecnológica y Educación para el Desarrollo (CITTED) y en el Centro Agrícola Cantonal de San Carlos Ubicado el Florencia.

\section{Materiales e instrumentos}

- Se utilizó suelo analizado del orden ultisol

- Se utilizaron 72 semillas de pasto Piatá (Brachiaria brizantha cv. Piatá)

- Se aplicaron 500 gramos de rastrojo de Crotalaria spectabilis Roth
- Se usaron estacas de madera para la confección de las parcelas

- Se usó un barreno para el muestreo de suelo al inicio y final

- Se utilizó un palin para tomar las muestras de suelo.

- Se usaron bolsas herméticas para recolectar las muestras de suelo y foliares

- Se usó una balanza digital para el pesaje de las muestras foliares y de los rastrojos incorporados.

\section{Metodología}

Para los ensayos se utilizó el suelo superficial previamente analizado (Ah, 0-20 cm), semillas de pasto Piatá (Brachiaria brizantha $c v$. Piatá) y rastrojo de Crotalaria (Leguminoceae: Crotalaria spectabilis Roth.), marcada con urea ${ }^{15} \mathrm{~N}$, y con un exceso de átomos de un $10 \%$.

Las semillas de crotalaria se sembraron al voleo en un área de 10 x $10 \mathrm{~m}$ en el Centro de Investigaciones CITTED/UNED La Perla, las cuales germinaron a los 15 días. A los 22 días después de la siembra (dds), fueron marcadas con urea ${ }^{15} \mathrm{~N}$, para lo cual se procedió a realizar una dilución en la que se mezcló suelo con el isótopo, agregando poco de agua para hacer la mezcla, la cual fue aplicada a cada planta, realizando una semi luna a unos $5 \mathrm{~cm}$ de cada plántula y posteriormente tapando la mezcla para evitar que se lavara o afectara a las plántulas. Durante la época de floración (60dds), se cosechó la crotalaria, se picó finamente y fue secada al aire.

Por otro lado, se confeccionaron cuatro parcelas de $1 \mathrm{~m}^{2}$ cada una. Cada parcela correspondió a un tratamiento que contó con 18 semillas en total, distribuidas en tres hileras (tratamiento por triplicado), cada una de éstas contenía tres puntos de siembra con 2 semillas por hueco y separados cada $30 \mathrm{~cm}$, dejando de borde $10 \mathrm{~cm}$. Los tratamientos consistieron en la aplicación de rastrojo de Crotalaria en dosis diferentes, donde T1: aplicación de $1 \mathrm{~g}$ por hueco sembrado, T2: aplicación de $5 \mathrm{~g}$ por hueco sembrado, T3: aplicación de $10 \mathrm{~g}$ por hueco sembrado y T4: sin aplicación de rastrojo (tratamiento testigo). 
Entre las variables analizadas en la investigación están las siguientes: características fisicoquímicas del suelo de partida y final, e incremento del contenido de materia orgánica de suelo.

\section{Resultados}

\section{Características químicas del suelo durante los meses de desarrollo del pasto Piatá (Brachiaria brizantha cv. BRS Piatá)}

En el suelo del tratamiento final se encontraron incrementos en los niveles de nutrientes, siendo estadísticamente significativos los valores en el suelo final, comparado con la nutrición presentada en el suelo inicialmente, los principales aumentos que se dieron fueron por ejemplo en los elementos mayores como el N, Potasio (K), aunque en el contenido de nitrógeno aumento al final sigue teniendo un valor por debajo bajo al valor de referencia (Tabla 1), también se observa una reducción en el contenido de materia orgánica y carbono orgánico. Se presentó un aumento considerable en la concentración y mineralización de elementos como Calcio (Ca), Magnesio $(\mathrm{Mg})$, Cobre $(\mathrm{Cu})$, el $\mathrm{pH}$ se mantuvo oscilando entre 5,03 al inicio y 5,16 al final, y bajando el índice de acidez en 0,04 cmol (+) / L comparado con la presentada al inicio del ensayo. La concentración de micronutrientes como Zinc

TABLA 1

Características químicas del suelo cultivado con pasto Piatá en Florencia, San Carlos 2016.

\begin{tabular}{|c|c|c|c|c|c|c|c|}
\hline \multirow{2}{*}{ Variable } & \multirow{2}{*}{ Unidad } & \multicolumn{3}{|c|}{ Valores } & \multicolumn{2}{|c|}{ Control } & \multirow{2}{*}{ Referencia* } \\
\hline & & $\mathrm{T} 1$ & $\mathrm{~T} 2$ & T3 & T. inicial & T. final & \\
\hline $\mathrm{pH}\left(\mathrm{H}_{2} \mathrm{O}\right)$ & \multirow{10}{*}{ 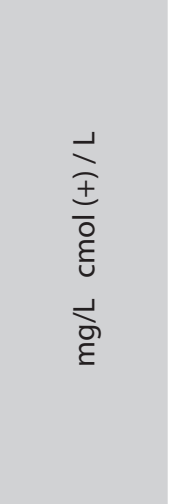 } & $5,16 \mathrm{a}$ & $5,16 a$ & $5,20 \mathrm{a}$ & $5,03 a$ & $5,16 \mathrm{a}$ & $5,4-6,5$ \\
\hline Acidez & & $0,18 \mathrm{a}$ & $0,20 \mathrm{a}$ & $0,55 \mathrm{a}$ & $0,30 \mathrm{a}$ & $0,26 \mathrm{a}$ & $<0,30$ \\
\hline $\mathrm{Ca}$ & & $8,40 \mathrm{~b}$ & $7,76 \mathrm{a}$ & $9,26 c$ & $7,07 \mathrm{a}$ & $9,10 \mathrm{c}$ & $4-20$ \\
\hline Mg & & $3,50 \mathrm{~b}$ & $3,13 \mathrm{a}$ & $5,13 d$ & $2,93 \mathrm{a}$ & $3,80 \mathrm{c}$ & $1-5$ \\
\hline K & & $0,43 a$ & $0,74 \mathrm{c}$ & $0,63 \mathrm{~b}$ & $0,52 \mathrm{a}$ & $0,60 \mathrm{~b}$ & $0,2-0,8$ \\
\hline$P$ & & $14,00 \mathrm{a}$ & $18,33 \mathrm{~b}$ & $22,33 \mathrm{c}$ & $26,00 \mathrm{a}$ & $19,30 \mathrm{~b}$ & $10-50$ \\
\hline $\mathrm{Zn}$ & & $2,33 \mathrm{a}$ & $2,26 a$ & $2,23 \mathrm{a}$ & $2,30 \mathrm{a}$ & $2,53 \mathrm{~b}$ & $10-50$ \\
\hline Mn & & $22,00 \mathrm{~b}$ & $22,66 \mathrm{~b}$ & $11,33 \mathrm{a}$ & $95,00 \mathrm{a}$ & $43,30 \mathrm{c}$ & $10-50$ \\
\hline $\mathrm{Cu}$ & & $8,66 \mathrm{a}$ & $9,00 \mathrm{a}$ & $9,33 \mathrm{a}$ & $8,67 \mathrm{a}$ & $9,66 \mathrm{a}$ & $1-20$ \\
\hline $\mathrm{Fe}$ & & $96,00 \mathrm{a}$ & $114,00 \mathrm{~b}$ & $159,00 \mathrm{c}$ & $102,70 \mathrm{a}$ & 99,33 a & $10-50$ \\
\hline N & \multirow{2}{*}{$\%$} & $0,13 \mathrm{c}$ & $0,11 \mathrm{a}$ & $0,12 \mathrm{~b}$ & $0,11 \mathrm{a}$ & $0,13 \mathrm{c}$ & $0,2-0,7$ \\
\hline C & & $1,21 \mathrm{c}$ & $1,22 d$ & $1,05 \mathrm{C}$ & $0,79 a$ & $0,64 a$ & $1-2,5$ \\
\hline $\mathrm{C} / \mathrm{N}$ & - & 9,30 & 11,09 & 8,75 & 7,18 & 4,92 & 10 \\
\hline \multicolumn{8}{|c|}{ Relaciones catiónicas } \\
\hline $\mathrm{Ca} / \mathrm{Mg}$ & & 2,40 & 2,48 & 1,80 & 2,41 & 2,39 & $2-5$ \\
\hline $\mathrm{Ca} / \mathrm{K}$ & & 19,53 & 10,48 & 14,19 & 13,60 & 15,16 & $5-25$ \\
\hline $\mathrm{Mg} / \mathrm{K}$ & & 8,14 & 4,23 & 8,14 & 5,63 & 6,33 & $2,5-15$ \\
\hline $\mathrm{Ca}+\mathrm{Mg}+\mathrm{K}$ & & 12,33 & 11,63 & 14,99 & 10,52 & 13,50 & $5-12$ \\
\hline$(\mathrm{Ca}+\mathrm{Mg}) / \mathrm{K}$ & & 27,67 & 14,72 & 10,66 & 19,23 & 21,50 & $10-40$ \\
\hline SA & $\%$ & 1,44 & 1,69 & 3,53 & 2,77 & 1,89 & $<10$ \\
\hline CICE & $\mathrm{cmol}(+) / \mathrm{L}$ & 12,51 & 11,83 & 15,57 & 10,82 & 13,76 & $5-25$ \\
\hline
\end{tabular}

Nota: *Méndez y Bertsch (2012). SA: saturación de acidez, CICE: capacidad de intercambio efectiva. Tratamientos (1 m²) =T1: $1 \mathrm{~g} / \mathrm{planta}$, T2: $5 \mathrm{~g} /$ planta, T3: $10 \mathrm{~g} /$ planta, T Control: $0 \mathrm{~g} /$ planta. Medias con una letra común no son significativamente diferentes ( $p>0,05)$, no hubo prueba de significancia en las relaciones catiónicas. 
$(\mathrm{Zn})$, Cobre $(\mathrm{Cu})$ y Hierro $(\mathrm{Fe})$, mantuvieron sus niveles durante el desarrollo del pasto Piatá (Brachiaria brizantha cv. BRS Piatá), siendo el Zn el que presenta deficiencia en el suelo tanto al inicio $(2,30 \mathrm{mg} / \mathrm{L})$ como al final $(2,53 \mathrm{mg} / \mathrm{L}) \mathrm{del}$ ensayo ya que posee un contendido $<10 \mathrm{mg} / \mathrm{L}$ ,en el caso del Fe este elemento menor posee un exceso característica de los suelos ultisoles, aun así se observa una disminución de 3,33 mg/L de Fe comparado con el contenido en el suelo inicial Otra deficiencia que se presenta en el suelo de acuerdo al cuadro 5, es el contenido de carbono orgánico en el suelo, el cual se encuentra por debajo del $1 \%$.

En cuanto a las relaciones catiónicas presentadas por el suelo, se observa que hay un aumento en la relación de estas bases, con respecto al análisis químico de suelos realizado al inicio, las diferencias más marcada son entre las relaciones catiónicas de $\mathrm{Ca}+\mathrm{Mg}+\mathrm{K}$ y $(\mathrm{Ca}+\mathrm{Mg}) / \mathrm{K}$, en el caso de la primera relación al inicio tenía un valor de 10,52 y al final paso a 13,50, en el caso de la relación $(\mathrm{Ca}+\mathrm{Mg}) / \mathrm{K}$ tuvo un aumento de 2,27 con respecto al muestreo inicial. La CICE también en este caso se vio favorecida ya que hubo un aumento de 2,94 cmol (+) / L, comparado con la CICE presentada por el suelo al inicio, un aspecto importante fue la reducción de la acidez la cual paso de $0,30 \mathrm{cmol}(+) / \mathrm{L}$ al inicio, a 0,26 cmol (+) / L, aunque según el análisis de varianza no existe diferencia estadísticamente significativa $\mathrm{p}>0,05$.

El pH es ácido no habiendo diferencia, la acidez presentada es ligeramente baja, pero no hay problemas de acidez, a pesar de presentar valores bajos en el complejo de cambio catiónico, no hubo diferencia en las relaciones catiónicas, salvo el contenido de Manganeso (Mn), cuyos valores bajan significativamente al finalizar el desarrollo del pasto. En cuanto al incremento de nitrógeno que hubo en el suelo con respecto al inicio es de 0,02 puntos, que corresponde a un $15 \%$.

En la figura 1, se puede observar que la leguminosa Crotalaria spectabilis Roth, como abono verde durante el establecimiento de la pastura Piatá (Brachiaria brizantha cv BRS Piatá), tuvo un excelente efecto ya que sus niveles de nitrógeno se vieron incrementados con respecto a los análisis de suelos que se llevaron a cabo al inicio, en el caso específico del nitrógeno hubo un incremento de un $15 \%$.

En los valores de Carbono en el suelo, se observa que se da una disminución, en 0,16 puntos, es decir un $20 \%$ menos que el contenido que había en el suelo inicial, esto debido al proceso de mineralización.

TABLA 2

Aporte de materia orgánica al suelo de las diferentes dosis de rastrojo de Crotalaria spectabilis Roth.

\begin{tabular}{cc} 
Tratamiento* & Contenido de $\mathrm{MO}(\%)^{* *}$ \\
T1 & $2,09 \mathrm{c}$ \\
$\mathrm{T} 2$ & $2,11 \mathrm{~d}$ \\
$\mathrm{~T} 3$ & $1,81 \mathrm{~b}$ \\
Testigo & $1,11^{\mathrm{a}}$ \\
\hline
\end{tabular}

Nota: * Tratamientos (1m2) = T1: $1 \mathrm{~g} /$ planta, T2: 5 g/planta, T3: 10 $\mathrm{g} /$ planta, T Control: $0 \mathrm{~g} /$ planta. Medias con una letra común no son significativamente diferentes $(p>0,05)$.

** MO (Materia orgánica).

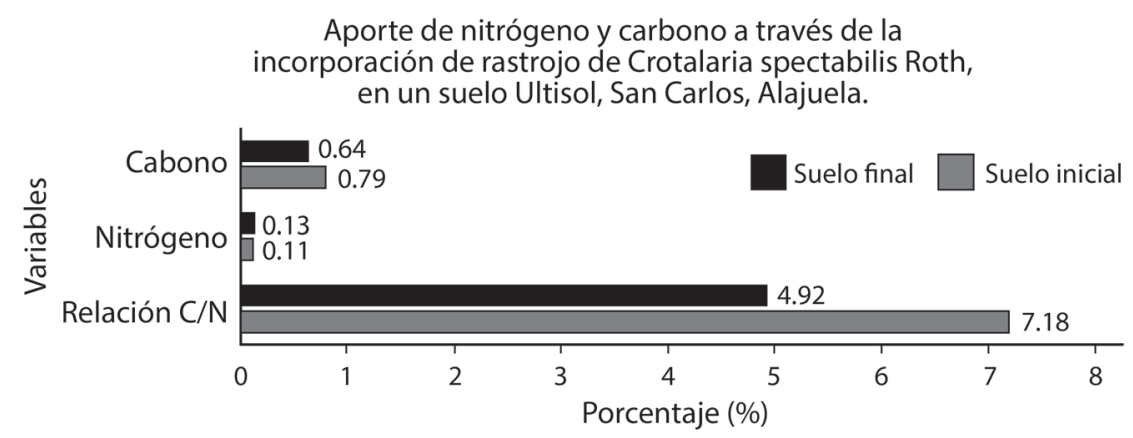

Figura 1. Aporte de nitrógeno y carbono Crotalaria spectabilis Roth, en un suelo Ultisol, San Carlos, Alajuela. 
En los tres tratamientos en los que se utilizó rastrojo de Crotalaria spectabilis Roth, se obtuvo un incremento que fluctuó entre un $38,67 \%$ y un $47,39 \%$, comparado al tratamiento testigo.

De acuerdo con la Tabla 2 se puede observar que hay diferencia estadísticamente significativa $(\mathrm{p}<0,05)$, entre los tratamientos durante los días de evaluación.

TABLA 3

Análisis Fisicoquímico del abono verde de Crotalaria spectabilis Roth aplicado.

\begin{tabular}{ccccc} 
Variable & Unidad & \multicolumn{3}{c}{ Valores } \\
\cline { 3 - 4 } $\mathrm{Ca}$ & & 1,07 & 2,11 & 2,17 \\
$\mathrm{Mg}$ & & 0,15 & 0,25 & 0,23 \\
$\mathrm{~K}$ & & 0,93 & 1,85 & 1,61 \\
$\mathrm{P}$ & ఏ & 0,41 & 0,24 & 0,26 \\
$\mathrm{Zn}$ & शे & 31,33 & 50,33 & 41,55 \\
$\mathrm{Mn}$ & ह & 44,00 & 85,78 & 82,22 \\
$\mathrm{Cu}$ & & 12,44 & 18,33 & 16,89 \\
$\mathrm{Fe}$ & & 143,00 & 163,33 & 158,89 \\
$\mathrm{~N}$ & & 2,53 & 2,75 & 4,04 \\
$\mathrm{C}$ & $\%$ & 31,91 & 32,54 & 42,89 \\
$\mathrm{C} / \mathrm{N}$ & - & 11,12 & 9,93 & 10,89 \\
\hline
\end{tabular}

Nota: * Tratamientos $=\mathrm{T} 1: 1 \mathrm{~g}$ de rastrojo de Crotalaria spectabilis Roth, T2: $5 \mathrm{~g}$ de rastrojo de Crotalaria spectabilis Roth, T3: $10 \mathrm{~g}$ de rastrojo de Crotalaria spectabilis Roth.

Como se observa en la Tabla 3, el abono verde o rastrojo de Crotalaria spectabilis Roth, es una excelente opción para mejorar la nutrición de los suelos y mejorar la disponibilidad de nutrimentos en el mismo, este abono presenta niveles importantes en macronutrimentos como Carbono (C) principalmente y Nitrógeno $(\mathrm{N})$, otros elementos de alto contenido son $\mathrm{Fe}, \mathrm{Zn}$ y Mn.

El porcentaje promedio de nitrógeno de las muestras del rastrojo de Crotalaria spectabilis Roth de 1 gramo, analizadas a la hora de aplicar el abono verde fue de 2,53\%. Para las muestras de 5 gramos su promedio fue 2,75 y para las muestras analizadas de 10 gramos contenía un promedio de 4,01 de $\mathrm{N}$, el promedio general de nitrógeno fue de $3,09 \%$.

El contenido de carbono orgánico de los rastrojos de Crotalaria spectabilis Roth, de 1, 5 y 10 gramos aplicados durante la siembra del pasto Piatá (Brachiaria brizantha cv. BRS Piatá), fueron de la siguiente manera: las muestras de 1 gramo oscilo, con un promedio de 31,91 . Para las muestras de $5 \mathrm{~g}$, su promedio fue de 32,54 y para las muestras analizadas de $10 \mathrm{~g}$, sus porcentajes en promedio eran de 42,89 , el promedio general de carbono orgánico fue de $35,78 \%$

La relación $\mathrm{C} / \mathrm{N}$ que existen en los rastrojos de crotalaria (Crotalaria spectabilis Roth) de 1 gramo en promedio era de 11,12. Para las muestras de $5 \mathrm{~g}$, su promedio fue 9,93 y para las muestras analizadas de $10 \mathrm{~g}$ tenían un promedio de 10,89 , la media general de la relación $\mathrm{C} / \mathrm{N}$ fue de 10,65 .

\section{Discusión}

Como se observó en la Tabla 1, el terreno utilizado para las parcelas experimentales presentó valores bajos de $\mathrm{pH}$ y alta acidez, posiblemente con problemas de toxicidad por aluminio intercambiable. Estas condiciones son comunes en ultisoles, donde se observa que la fertilidad química del suelo es baja y es alta la presencia de hierro en forma oxidada. En cuanto al complejo de cambio, los valores están dentro del rango normal, inclusive las relaciones catiónicas, la saturación de acidez y la capacidad de intercambio, por lo que no hay un desbalance de los principales cationes esenciales.

Los anteriores valores nutricionales sirven de punto de partida para conocer el estado de fertilidad química del suelo, pero es necesario conocer la disponibilidad de nutrimentos tanto en el suelo como en el material vegetal que se aplica como abono verde, especialmente de $\mathrm{N}$ y la relación carbono nitrógeno $(\mathrm{C} / \mathrm{N})$ (Tabla 1$)$.

A través de los resultados del análisis de suelo se puede interpretar que existe un alto contenido de Fe y Mn con respecto a los valores de referencia que indica Molina y Bertsch (2012), lo cual puede ocasiono que se presentara una reducción del hierro, siendo una forma menos 
soluble y provocando toxicidad del pasto, y de esta manera afectando el crecimiento del forraje, debido a que el pasto absorbe de manera indiscriminada este elemento, provocando lesiones en su raíz, follaje, así como los meristemos de crecimiento, y además el bloqueo de la absorción del macronutriente Potasio.

De acuerdo con los resultados obtenidos apartir del análisis de suelo realizado por el Instituto Nacional de Innovación y Trasferencia en Tecnología Agropecuaria (INTA), se observa que en el complejo de bases de intercambio catiónico tanto el $\mathrm{Ca}, \mathrm{Mg}$ y $\mathrm{K}$ tuvieron una presencia bastante importante de sus valores, a excepción como se observa en la Tabla 1, donde en el tratamiento 3 hay un desbalance catiónico entre las bases de $\mathrm{Ca} / \mathrm{Mg}$, debido a la mayor cantidad de calcio con respecto al magnesio. Según Arias (2015), en su investigación menciona que cuando los suelos presentan altas concentraciones específicamente de Ca y $\mathrm{Mg}$, se relaciona con una mejor y alta fertilidad del suelo en estudio.

En lo que respecta a los valores de acidez, no existen problemas ya que se encuentra en un rango de 5,03 a 5,2 coincidiendo con los valores de referencia indicados por (Meléndez y Molina, 2001) se puede observar mediante el análisis de suelo que la acidez intercambiable es óptima, en las diferentes parcelas, donde se aplicó el abono verde por lo que la acidez no es una limitante para el crecimiento y desarrollo del pasto Piatá (Brachiaria brizantha cv. BRS Piatá) que soporta hasta valores de $\mathrm{pH}$ de 4,5.

De acuerdo con los niveles porcentuales de nitrógeno, carbono orgánico y materia orgánica mencionados por Nuñez (2001) se puede interpretar que los niveles de contenido presentes en el suelo analizado se encuentran en un nivel bajo, lo cual resulta normal en un ultisol y bajo las condiciones del sitio experimental. Estas características se pueden deber a que los valores de materia orgánica, su composición química, la cantidad de biomasa aportada al suelo, velocidad de mineralización y humificación, dependen de condiciones ecológicas específicas y de las condiciones fisicoquímicas del suelo (Nuñez, 2001), mejorado un poco más al final gracias al aporte del rastrojo de Crotalaria spectabilis Roth.
Se observó que tanto el suelo como el análisis foliar de Crotalaria presentan valores óptimos de humificación, por lo que es de esperar una buena nutrición del pasto; esto último dependerá no solo de la disponibilidad de nitrógeno sino también de las condiciones agroclimáticas donde se desarrolle, además de valorar la proporción en carbono y en nitrógeno como dos variables independientes.

Fundamentalmente la relación $\mathrm{C} / \mathrm{N}$ en un abono verde varía según la especie y la edad de la planta, es considerada como buen indicador de la susceptibilidad de la hojarasca a ser degradada y, según el estudio de (Jiménez, A., Farfán, F, \& Morales, C. 2005), en el caso de que los residuos sean altos en carbono y bajos en nitrógeno, la descomposición será lenta, en el caso contario se transformaría en amonio, lo que implica una disminución en la actividad biológica involucrada en la mineralización de nutrimentos esenciales. De esta manera se puede interpretar que el abono verde que se aplicó posee una descomposición lenta la cual ocurrió aproximadamente a los 20 días.

Según la Figura 1, se observa que el contenido de carbono final es menor que el presente que había al inicio, eso se debe al proceso de mineralización, en donde, los microorganismos presentes en el suelo van a convertir el material orgánico del suelo (rastrojo de Crotalaria spectabilis Roth), a una forma inorgánica, de esta manera mejorando la fertilidad del recurso edáfico. La tasa y alcance de la mineralización depende de la disponibilidad de oxígeno lo que se concluye que en este suelo Ultisol de la zona Norte tenía una buena presencia de oxígeno, requisito indispensable en este tipo de abonos para que tengan una buen degradación y liberación de nutrimentos.

Con respecto a la relación Carbono Nitrógeno $(\mathrm{C} / \mathrm{N})$, es un factor que influye sobre la velocidad de descomposición de la materia fresca, entonces como se observó en la Figura 1, la relación $\mathrm{C} / \mathrm{N}$, se disminuyó debido a que existe una mayor descomposición, ya que de pierde Carbono en forma gaseosa $\left(\mathrm{Co}_{2}\right)$, mientras tanto el $\mathrm{N}$ permanece en combinaciones orgánicas. 
Como se pudo observar el efecto de la incorporación de rastrojo de Crotalaria spectabilis Roth, fue positivo teniendo en promedio un aumento del $47 \%$ en el contenido de materia orgánica, con respecto al tratamiento testigo. Este aumento permitió mejorar la aireación en el suelo, mayor retención de la humedad en el recurso edáfico, así como el mejoramiento de las propiedades químicas como el reciclaje de nutrimentos: nitrógeno, fósforo, azufre, manganeso, zinc del suelo (Kass, 2007).

Gracias al incremento en el valor de materia orgánica presente en el suelo, aumentó la capacidad de intercambio catiónico, mejoramiento en el contenido nutricional del suelo debido al proceso de mineralización de la materia orgánica. Es importante mencionar que este aumento permitió una mayor actividad de los microorganismos presentes en el suelo, sirviendo como sustrato y beneficiando el buen desarrollo del recurso suelo.

Como se observó en la Tabla 2 y 3, los residuos orgánicos son una fuente importante de nitrógeno. Debido a que libera compuestos que, por procesos de nitrificación en suelos bien drenados, conduce a la formación de nitratos. También es fuente de azufre (S) y fósforo (P); a través de los procesos que genera ruptura y posterior descomposición de materia orgánica (Nuñez, 2006).

Es importante mencionar que el reciclaje de la materia orgánica no solo involucra a elementos como S y P, sino también hay aporte de macronutrientes como el Calcio ( $\mathrm{Ca}$ ), Magnesio $(\mathrm{Mg})$ y Potasio (K), que en conjunto forman una interrelación con la planta, clima, y hace que el aporte sea más importante.

\section{Conclusiones y Recomendaciones}

El aporte de rastrojos de Crotalaria spectabilis Roth, como abono incorporado al pasto Piatá (Brachiaria brizantha cv. BRS Piatá), incrementó en un $15 \%$ el contenido de nitrógeno del suelo y un $47 \%$ en el contenido de materia orgánica.

Las diferencias existentes en las propiedades químicas de suelo se deben a la composición nutricional de las diferentes dosis de abono verde incorporadas, en las cuales se logró un mejoramiento de estas propiedades específicamente en el contenido de N, C, contenido de materia orgánica y la mayoría de los elementos menores, comparado con las características de suelo inicial.

Se deben realizar estudios sobre la degradación de la leguminosa de Crotalaria spectabilis Roth, con el fin de conocer cuál es el momento en que se da la mayor liberación de nitrógeno y nutrientes al suelo e incorporarlo antes al suelo para un mejor aprovechamiento.

No se debe solo utilizar material incorporado al suelo como abono verde, si no también utilizar la leguminosa como planta acompañante del cultivo principal, pasto Piatá (Brachiaria brizantha $c v$. BRS Piatá), para aprovechar la fijación biológica de nitrógeno de esta especie.

En general se puede decir que gracias al proceso de degradación y mineralización que sufren las leguminosas utilizadas en los diferentes ensayos, permite un enriquecimiento nutricional de forma natural a largo plazo, mejorando de esta forma las propiedades físicoquímicas del suelo, así como la actividad microbiológica del mismo.

La dinámica de mineralización y descomposición del rastrojo de Crotalaria spectabilis Roth, fue regulado en gran parte por factores bióticos y abióticos, provocando una liberación de nutrientes más lenta cuando se utilizó lo mínimo en cantidad de rastrojo, comparado a los demás tratamientos.

\section{Bibliografía Consultada}

Araya, K. (2014). Efecto del Arachis y la fertilización nitrogenada sobre la producción y composición botánica en pasturas de Brachiaria brizantha $\mathrm{c} v$. Toledo $y$ Brachiaria Híbrido cv. Mulato. (Tesis de pregrado). Instituto Tecnológico de Costa Rica. San Carlos, Costa Rica.

Arias, A. (2015). Disponibilidad de nitrógeno en el rastrojo de Gandul (Cajanus cajan), bajo ambiente controlado en San Isidro de Heredia. (Tesis de pregrado). Universidad Estatal a Distancia, San José, Costa Rica.

Durán, H. (2015). Políticas ambientales y desarrollo sustentable: El contexto de su aplicación. Santiago, 
Chile. Recuperado de http://www.cepal.org/publicaciones/xml/6/4496/duran.htm

Jiménez, A., Farfán, F, \& Morales, C. (2005). Biomasa seca y contenido de nutrientes de Cajanus cajan Crotalaria juncea y Tephrosia candida empleadas como abonos verdes en cafetales. Cenicafé 56(2), 93-109.

Kass, D. (2007). Fertilidad de Suelos. San José: EUNED.

Nuñez, J. (2001). Fundamentos de Edafología. San José: EUNED.
Nuñez, J. (2006). Manual de Laboratorio de Edafología. San José: EUNED.

Paredes, M.C. 2013. Fijación biológica de nitrógeno en leguminosas y gramíneas. (Tesis de grado). Universidad Católica de Argentina. Buenos Aires, Argentina.

Ramírez, J. L., Herrera, R. S., Leonard, I., Verdecia, D., \& Álvarez, Y. (2012). Rendimiento y calidad de la Brachiaria decumbens en suelo fluvisol del Valle del Cauto, Cuba. REDVET. Revista Electrónica de Veterinaria, 13(4), 1-11. 